\title{
ON THE THEORY OF THE TETRAHEDRON
}

\author{
N. A. COURT
}

I. Definition. We associate with the general tetrahedron $(T)$ $=A B C D$ a sphere $(Q)$ whose center is the Monge point $M$ of $(T)$ and the square of whose radius is

$$
q^{2}=\left(M O^{2}-K^{2}\right) / 3,
$$

where $O$ and $R$ are the center and the radius of the circumsphere $(O)$ of $(T)$.

In what follows, a number of propositions regarding the sphere $(Q)$ will be established and it will be shown that from the properties of $(Q)$ may be derived, as special cases, properties of the polar sphere $(H)$ of the orthocentric tetrahedron $\left(T_{h}\right)$.

For want of a better name we shall refer to $(Q)$ as the "quasi-polar" sphere of the general tetrahedron $(T)$.

The expression $M O^{2}-R^{2}$ is the power of the Monge point $M$ of $(T)$ for the sphere $(O)$.

THEOREM 1. The square of the radius of the quasi-polar sphere of the general tetrahedron is equal to one-third of the power of the Monge point of the tetrahedron for its circumsphere.

The sphere $(Q)$ is real, a point sphere, or imaginary according as $M O$ is greater than, equal to, or smaller than $R$. Moreover, we have $M O<2 R$, for the mid-point of $M O$ is the centroid $G$ of $(T)$, and $G$ necessarily lies within the sphere $(O)$.

Corollary. In an orthocentric tetrahedron $\left(T_{h}\right)$ the Monge point coincides with the orthocenter $H$, and the above properties of $(Q)$ are valid for the polar sphere $(H)$ of $\left(T_{h}\right){ }^{1}$

The Monge point $M$ of $(T)$ is a center of similitude of the circumsphere $(O)$ and the twelve point sphere $(L)$ of $(T),{ }^{2}$ hence $M$ is the center of a sphere of antisimilitude of $(O)$ and $(L)$, that is, a sphere with respect to which the spheres $(O)$ and $(L)$ are inverse of one another.

Presented to the Society, December 31, 1941; received by the editors November 22, 1941.

${ }^{1}$ Nathan Altshiller-Court, Modern Pure Solid Geometry, New York, 1935, p. 265, $\S 813$. This book will be referred to as MPSG.

${ }^{2}$ MPSG, p. 251, $\$ 764$. 
The line of centers $L O$ of the spheres $(L),(O)$ meets $(O)$ in the points whose distances from $M$ are $M O-R$ and $M O+R$. Again, the line $L O$ meets $(L)$ in two points whose distances from $M$ are $M L-R / 3$ and $M L+R / 3$, for the radius of $(L)$ is equal to $R / 3$. Now the points $M O+R, M L-R / 3$ correspond to each other in the inversion considered (and so do the points $M O-R, M L+R / 3$ ), hence if $x^{2}$ is the constant of inversion, we have

$$
\begin{aligned}
x^{2} & =(M O+R)(M L-R / 3)=(M O+R)(3 M L-R) / 3 \\
& =\left(M O^{2}-R^{2}\right) / 3=q^{2} .
\end{aligned}
$$

But the constant of inversion is the square of the radius of the sphere of inversion, and the sphere of inversion is coaxial with the two inverse spheres.

THEOREM 2. The quasi-polar sphere of the general tetrahedron is coaxial with the circumsphere and the general twelve point sphere of the tetrahedron.

Corollary. To the square $(L)$ of $(T)$ corresponds in the orthocentric tetrahedron $\left(T_{h}\right)$ the second twelve point sphere, and from the above property of $(Q)$ we obtain the known property of the polar sphere of $\left(T_{h}\right) .^{3}$

THEOREM 3. The sum of the powers of the vertices of a general tetrahedron with respect to the quasi-polar sphere of that tetrahedron is equal to one-third of the sum of the squares of the edges of the tetrahedron.

The power $D_{q}$ of the vertex $D$ of $(T)$ for the sphere $(Q)$ is

$$
D_{q}=M D^{2}-q^{2}=M D^{2}-\left(M O^{2}-R^{2}\right) / 3,
$$

and from the triangle $D M O$ we have

$$
D M^{2}+D O^{2}=2 D G^{2}+M O^{2} / 2=2 D G^{2}+2 O G^{2},
$$

hence

$$
D_{q}=2 D G^{2}+2\left(O G^{2}-R^{2}\right) / 3 .
$$

Adding to $D_{q}$ the analogous formulas relative to the vertices $A, B, C$ of $(T)$ we obtain

$$
A_{q}+B_{q}+C_{q}+D_{q}=2\left(A G^{2}+B G^{2}+C G^{2}+D G^{2}\right)+8\left(O G^{2}-R^{2}\right) / 3 .
$$

Now if we denote by $k^{2}$ the sum of the squares of the edges of $(T)$, we have

\footnotetext{
${ }^{3}$ MPSG, p. 264, §805.
} 


$$
\begin{gathered}
A G^{2}+B G^{2}+C G^{2}+D G^{2}=k^{2} / 4,^{4} \\
O G^{2}-R^{2}=-k^{2} / 16,{ }^{5}
\end{gathered}
$$

hence

$$
A_{q}+B_{q}+C_{q}+D_{q}=k^{2} / 2-k^{2} / 6=k^{2} / 3 .
$$

COROLLARY. In an orthocentric tetrahedron the sum of the squares of a pair of opposite edges is constant, hence the sum of the powers of the vertices of the tetrahedron for the polar sphere is equal to the sum of the squares of a pair of opposite edges. ${ }^{6}$

ThEOREM 4. The sum of the squares of the distances of the Monge point of a tetrahedron from the mid-points of the edges is equal to three times the sum of the squares of the radii of the circumsphere and the quasi-polar sphere of the tetrahedron.

Let $E, F$ be the mid-points of the edges $D A, B C$ of $(T)$. The midpoint of the bimedian $E F=m_{a}$ of $(T)$ is the centroid $G$ and therefore we have, from the triangle $M E F$,

$$
M E^{2}+M F^{2}=2 M G^{2}+E F^{2} / 2=2 O G^{2}+m_{a}^{2} / 2 .
$$

We have two analogous formulas relative to the other two pairs of opposite edges of $(T)$. Adding the three relations we have

$$
\sum M E^{2}=6 O G^{2}+\left(m_{a}^{2}+m_{b}^{2}+m_{c}^{2}\right) / 2 .
$$

Now the expression in the parenthesis is equal to ${ }^{7} k^{2} / 4$, hence, making use of the formula (b), we have

$$
\sum M E^{2}=6\left(R^{2}-k^{2} / 16\right)+k^{2} / 8=3 R^{2}+3\left(R^{2}-k^{2} / 12\right) .
$$

But, eliminating $O G$ between the formulas (a) and (b) we have

$$
R^{2}-k^{2} / 12=q^{2},
$$

hence

$$
\sum M E^{2}=3\left(R^{2}+q^{2}\right) .
$$

COROLLARY. The proposition is valid for the orthocentric tetrahedron. ${ }^{8}$

THEOREM 5. The sum of the powers of the Monge point of a tetra-

${ }^{4}$ National Mathematics Magazine, vol. 15 (1941), p. 273, §3.

5 V. Thebault, Nouvelles Annales de Mathématiques, (4), vol. 19 (1919), p. 425.

${ }^{6}$ Mathesis, vol. 42 (1928), p. 338, \$2.

7 MPSG, p. 56, §186.

${ }^{8}$ MPSG, p. 275, Example 4. 
hedron with respect to the six spheres having for diameters the edges of the tetrahedron is equal to six times the square of the radius of the quasipolar sphere of the tetrahedron.

The power of the Monge point $M$ for the sphere $(B C)$ having for diameter the edge $B C=a$ of $(T)$ is equal to $M F^{2}-a^{2} / 4$. We have five analogous expressions relative to the other five edges of $(T)$. Adding the six expressions we have

$$
\sum M F^{2}-k^{2} / 4
$$

or, using the value of $\sum M F^{2}$ from the preceding article,

$$
6\left(R^{2}-k^{2} / 16\right)+k^{2} / 8-k^{2} / 4=6 q^{2} .
$$

In an orthocentric tetrahedron the spheres having for diameters the edges of the tetrahedron are orthogonal to the polar sphere.

COROLLARY. The power of the orthocenter for the six spheres is equal to the square of the radius of the polar sphere.

The powers of the Monge point of $(T)$ for the two spheres having for diameters a pair of opposite edges of $(T)$ are equal. ${ }^{9}$

THEOREM 6. The sum of the powers of the Monge point of a tetrahedron with respect to three spheres having for diameters three concurrent (or coplanar) edges of the tetrahedron is equal to three times the square of the radius of the quasi-polar sphere.

The centroid $G$ of $(T)$ bisects the segment $A A^{\prime}$ joining $A$ to the corresponding vertex $A^{\prime}$ of the tetrahedron $\left(T^{\prime}\right)$ twin to $(T)$, hence $A M A^{\prime} O$ is a parallelogram.

Let $A_{1}$ be the diametric opposite of $A$ on the circumsphere $(O)$ of $(T)$. The quadrilateral $M A^{\prime} A_{1} O$ is a parallelogram, for $O A_{1}$ and $M A^{\prime}$ are equal and parallel. Hence the diagonal $M A_{1}$ bisects the diagonal $O A^{\prime}$, and therefore meets the median $A^{\prime} G$ of the triangle $A^{\prime} O M$ in the centroid $G_{a}$ of that triangle. Thus

$$
G G_{a}=A^{\prime} G_{a} / 2=A G / 3,
$$

and $G_{a}$ is therefore the centroid of the face $B C D$ of $(T)$. Moreover $M G_{a}=M A_{1} / 3$.

Let $A_{2}$ be the second point of intersection, besides $A$, of the line $A M$ with the sphere $(O)$. The line $A_{1} A_{2}$ is perpendicular to the line $A M A_{2}$, hence if $F$ is the foot of the perpendicular from $G_{a}$ upon $A M A_{2}$ we have

${ }^{9}$ G. Gallucci, Nouvelles Annales de Mathématiques, (3), vol. 16 (1897), p. 17, §5. 


$$
M F: M A_{2}=M G_{a}: M A_{1}=1: 3,
$$

and therefore

$$
M A \cdot M A_{2}=3 M A \cdot M F \text {. }
$$

But $M A \cdot M A_{2}$ is the power of $M$ for the sphere $(O)$, hence by Theorem $1, M A \cdot M F$ is equal to the square of the radius of the quasi-polar sphere $(Q)$, that is, the points $A, F$ are inverse with respect to $(Q)$.

The median $A G_{a}$ of $(T)$ subtends a right angle at $F$, hence the sphere $\left(A G_{a}\right)$ having $A G_{a}$ for diameter is orthogonal to $(Q)$, similarly for the three analogous spheres $\left(B G_{b}\right),\left(C G_{c}\right),\left(D G_{d}\right)$. Thus this theorem follows:

THEOREM 7. The quasi-polar sphere of a tetrahedron is orthogonal to the four spheres having for diameters the four medians of the tetrahedron. ${ }^{10}$

Since the points $A, F$ are inverse for $(Q)$ (Theorem 7 ), the polar plane $\alpha$ of $A$ for $(Q)$ is perpendicular to $A M$ at $F$; hence the line $F G_{a}$ is the trace of $\alpha$ in the plane $A M G O$.

THEOREM 8. The polar reciprocal tetrahedron of a given tetrahedron $(T)$ with respect to the quasi-polar sphere of $(T)$ is circumscribed about the medial tetrahedron of $(T)$.

THEOREM 9. The faces of the polar reciprocal tetrahedron of a given tetrahedron $(T)$ with respect to the quasi-polar sphere of $(T)$ cut the spheres having for diameters the corresponding medians of $(T)$ along four circles lying on the same sphere, namely, the twelve point sphere of $(T)$.

The point $G_{a}$ lies on the twelve point sphere $(L)$ of $(T)$, and the diametric opposite of $G_{a}$ on $(L)$ lies on the line $A M ;^{11}$ hence the point $F$ also belongs to the sphere $(L)$. Thus the plane $\alpha$ (Theorem 8 ) cuts the two spheres $\left(A G_{a}\right)$ and $(L)$ along the same circle, and $F G_{a}$ is a diameter of that circle.

THEOREM 10. The faces of the polar reciprocal tetrahedron of the medial tetrahedron of a given tetrahedron $(T)$ with respect to the quasipolar sphere of $(T)$ cuts the spheres having for diameters the respective medians of $(T)$ along four circles lying on the same sphere, namely the circumsphere of $(T)$.

The polar plane of $G_{a}$ with respect to the quasi-polar sphere $(Q)$ passes through $A$ and is perpendicular to $M G_{a}$, hence the trace $K$

${ }^{10}$ American Mathematical Monthly, vol. 39 (1932), pp. 196, 197, §§11, 13.

${ }^{11}$ MPSG, p. 251. 
of this plane on $M G_{a}$ lies on the sphere $\left(A G_{a}\right)$ having for diameter the median $A G_{a}$ of $(T)$.

On the other hand, the line $M G_{a}$ passes through the diametric opposite $A_{1}$ of $A$ on the circumsphere $(O)$ of $(T)$, hence $K$ lies on $(O)$. Thus the line $A K$ is a common diameter of the two circles along which the polar plane considered cuts the two spheres $\left(A G_{a}\right)$ and $(O)$.

II. Definitions. We associate with the general tetrahedron $(T)$ the sphere having for center the centroid $G$ of $(T)$ and for the square of its diameter one-third of the sum of the squares of the bimedians of $(T)$. The sphere will be denoted by $(G)$ and referred to as the $G$-sphere of $(T)$. The sphere $(G)$ is concentric with the three spheres having for diametcrs the bimedians of $(T)$.

In an orthocentric tetrahedron the bimedians are equal, so that the three spheres having these bimedians for diameters coincide, and the $G$-sphere coincides with them, in the first twelve point sphere of the orthocentric tetrahedron, the three bimedians being diameters of that sphere.

THEOREM 1. The quasi-polar sphere and the $(G)$-sphere of the general tetrahedron are orthogonal.

The square of the radius, $g^{2}$, of the sphere $(G)$ is equal to ${ }^{7}(\$ \mathrm{I}$, Theorem 4)

$$
\left(m_{a}^{2}+m_{b}^{2}+m_{c}^{2}\right) / 3: 4=k^{2} / 48,
$$

hence

$$
q^{2}+g^{2}=\left(R^{2}-k^{2} / 12\right)+k^{2} / 48=R^{2}-k^{2} / 16=O G^{2}=M G^{2},
$$

that is, the square of the line of centers of the spheres $(Q),(G)$ is equal to the sum of the squares of their radii.

COROLLARY. The polar sphere and the first twelve point sphere of the orthocentric tetrahedron are orthogonal. ${ }^{12}$

THEOREM 2. The sphere $(G)$ belongs to the coaxial pencil formed by the circumsphere, the twelve point sphere, and the quasi-polar sphere of the tetrahedron.

The centroid $G$ is the second center of similitude, besides the point $M$, of the circumsphere $(O)$ and the twelve point sphere $(L)$ of $(T)$. Now the quasi-polar sphere $(Q)$ being a sphere of antisimilitude of

12 MPSG, p. 262, $\$ 799$. 
$(O)$ and $(L)$ ( $\$ I$, Theorem 2$)$, the sphere $(G)$ having $G$ for center and orthogonal to $(Q)$ ( $\$ I I$, Theorem 1$)$ is the second sphere of antisimilitude of $(O)$ and $(L)$; hence $(G)$ is coaxial with these spheres.

THEOREM 3. The four spheres having for centers the vertices of a tetrahedron and orthogonal to the quasi-polar sphere cut the spheres having for diameters the respective medians of the tetrahedron along four circles belonging to the same sphere, namely, the $(G)$-sphere of the tetrahedron.

The sphere $(A)$ having $A$ for center and orthogonal to the sphere $(Q)$ is coaxial with the spheres $(G)$ and $\left(A G_{a}\right)$, for the centers of these three spheres are collinear and all three are orthogonal to $(Q)$. Similarly for the vertices $B, C, D$ of $(T)$.

UNIVERSITY OF OKLAHOMA

\section{EUCLIDEAN CONCOMITANTS OF THE TERNARY CUBIC}

\section{T. L. WADE}

1. Introduction; construction of concomitants. In this paper we use the results of Cramlet [1] and the writer [2] to study the euclidean concomitants of the ternary cubic curve

$$
T_{a b c} X^{a} X^{b} X^{c}=0,
$$

where $a, b, c=1,2,3$ and $T_{a b c}$ is symmetric. With tensor algebra as the medium of investigation all types of concomitants are readily constructed, and their geometric interpretations are also readily made in most cases. As is conventional in classical invariant theory, the word concomitant will be used as meaning rational integral concomitant unless stated to the contrary.

As a consequence of Theorem 3 in [2], we have the following theorem.

TheOREM I. Every euclidean concomitant of the ground form $T_{a b c} X^{a} X^{b} X^{c}(a, b, c=1,2,3)$ is expressible by composition as a tensor of order zero with the use of the coefficient tensor $T_{a b c}$, the variable coordinate tensors $X^{a}$ and $U_{a}$, and the numerical tensors $\epsilon^{a b c}, L_{a}$, and $E^{a b}$.

Presented to the Society, September 5, 1941; received by the editors of the Transsctions of this Society, September 25, 1941; accepted by them and later transferred to this Bulletin. 